\title{
Vasovagal syncope with mild versus moderate autonomic dysfunction: a 13-year single-center experience
}

Han Eoul Lee, MD, Dong Won Lee, MD

Department of Pediatrics, Daegu Fatima Hospital, Daegu, Korea

Background: An adequate large-scale pediatric cohort based on nationwide administrative data is lacking in Korea.

Purpose: This study aimed to differentiate patients with VVS by autonomic dysfunction severity using the composite autonomic severity score (CASS) and compare the clinical manifestations and prognosis between patient subgroups.

Methods: We retrospectively reviewed the medical records of 66 VVS patients divided into 3 groups by CASS. To compare the differences between these groups, we analyzed VVS type, triggers, prodromal symptoms, management of syncope, and prognosis between patients with mild versus moderate autonomic dysfunction.

Results: Of our 66 patients with VVS, 41 had mild autonomic dysfunction (62.1\%) and 25 had moderate autonomic dysfunction (37.9\%). We found no significant intergroup differences in age, sex, inducible factor $(P=0.172)$, prodromal symptoms, laboratory findings, head-up tilt test, type of syncope, or prognosis $(P=0.154)$.

Conclusion: We found no evidence that autonomic dysfunction degree is affected by VVS characteristics, test findings, parameters, or prognosis; therefore, no further evaluations are needed to classify autonomic dysfunction severity.

Key words: Autonomic function tests, Composite autonomic severity score, Transcranial doppler, Vasovagal syncope

\section{Key message}

Question: It is well known that autonomic dysfunction contributes to vasovagal syncope (VVS). Does the degree of autonomic dysfunction contribute to clinical manifestations, diagnostic methods, treatment, and prognosis?

Finding: The clinical manifestations, diagnostic methods, treatment, and prognosis differ between patients with mild and moderate degrees of autonomic dysfunction.

Meaning: VVS is caused by autonomic dysfunction, but autonomic dysfunction severity need not be classified.

\section{Introduction}

Syncope is defined as a transient loss of consciousness (LOC) associated with cerebral hypoperfusion. ${ }^{1)}$ Syncope can happen when the cerebral blood flow is less than $30 \mathrm{~mL} / \mathrm{min}$ per $100 \mathrm{~g}$ brain tissue or when the mean cerebral blood flow rate is below $50 \% .{ }^{2,3)}$ It is a common problem in children and adolescents; approximately $15 \%$ of children and adolescents experience at least one episode of syncope before the age of $18 .^{4)}$ Syncope can be classified as neurally mediated, orthostatic, or cardiogenic. Among these, the most common form of syncope is vasovagal syncope (VVS) which is a subtype of neurally mediated syncope. ${ }^{5)}$

VVS can be diagnosed in approximately $50 \%$ of cases by detailed history taking and physical examination ${ }^{1}$; however, diagnosis is challenging for the remaining cases. A significant problem is that history taking is limited because patients lose consciousness, and so only bystanders can provide an account of events.

To maintain homeostasis in conditions of environmental change, vital signs are regulated by the autonomic nervous system. Stimulation from the sympathetic nervous system increases heart rate, and cardiac contractility, whereas that of the parasympathetic nervous system decreases heart rate, and cardiac contractility. To maintain adequate blood flow in the entire body, including brain, effective regulation of the autonomic nervous system is essential. ${ }^{6,7}$ In cases of VVS, not yet fully elucidated, decreased venous pooling by standing up rapidly or other reason, results in decrease in a venous return to the heart and diminishing cardiac output. Consequently, sympathetic nervous system increases to maintain blood pressure. The increased sympathetic nervous system has positive chronotropic and inotropic effects, makes ventricle contract more vigorously. But underfilled ventricle stimulates ventricular neural afferents to the brain. These afferents terminate in cardiovascular control centers where the input signals are integrated and results in sympathetic and parasympathetic activity to cardiovascular system. In VVS patients, activated central nuclei activate sympathetic tone and rapidly withdraw and increase in parasympathetic tone. Then increased parasympathetic tone reduces chronotropic response

Corresponding author: Dong Won Lee, MD. Department of Pediatrics, Daegu Fatima Hospital, 99 Ayang-ro, Dong-gu, Daegu 41199, Korea

凶Email: rabitover@hanmail.net, https://orcid.org/0000-0001-6907-8613

Received: 16 January, 2021, Revised: 21 April, 2021, Accepted: 24 May, 2021

This is an open-access article distributed under the terms of the Creative Commons Attribution Non-Commercial License (http://creativecommons.org/licenses/bync/4.0/) which permits unrestricted non-commercial use, distribution, and reproduction in any medium, provided the original work is properly cited.

Copyright (c) 2022 by The Korean Pediatric Society 
for evolving hypotension which is result of withdrawal of sympathetic tone. This response makes inappropriate heart rate slowing and the patients could LOC. 8 ,9)

The head-up tilt test (HUT) is widely used to diagnose VVS. The test elicits autonomic functional changes that provoke a syncope. However, a limitation of the test is that it is difficult to classify the severity of the autonomic dysfunction.

Thus, the hypothesis underlying our current study was that there would be differences in the type, nature, and test results of VVS in our study group depending on the degree of autonomic nerve dysfunction. And the purpose of this study, was to characterize and classify the degree of autonomic dysfunction using different autonomic function tests and compare the clinical features according to degree of autonomic dysfunction.

\section{Methods}

\section{Patient enrollment}

One hundred thrity-five patients diagnosis as syncope, aged between 8 to 18, admission to the Department of Pediatrics at Daegu Fatima Hospital between January 2008 and July 2020 were enrolled in this study and their medical records were retrospectively reviewed. We include all of these patients and we exclude limited available data, diagnosed as other than VVS, and negative HUT results.

Finally, we exclude 19 patients because of limited data, 20 with postural orthostatic tachycardia syndrome patients, 15 with negative HUT, 2 with brain tumors, 2 with encephalopathy, and 2 with atrioventricular block. After excluding these patients, we diagnose 66 patients with VVS and enrolled in our study. Of our VVS patients, we diagnosed mild autonomic dysfunction in 41 patients, moderate autonomic dysfunction in 25 , and no other patients were diagnosed as severe autonomic dysfunction.

\section{Data collection}

Patients' medical records were retrospectively reviewed to examine their age, sex, height, body weight, prodromal symptoms, and triggers. After admission, the patients underwent a blood test, which included complete blood count, total protein, albumin, serum electrolytes, aspartate aminotransferase, alanine aminotransferase, C-reactive protein, in addition to a thyroid function test, electrocardiogram, Holter test, chest x-ray, electroencephalogram (EEG), echocardiogram, HUT, Transcranial Doppler (TCD), and autonomic function tests. All patients in our study were followed-up through the outpatient department to analyze prognosis.

\section{HUT and TCD}

HUT (TT-800s, Pampas, Gimpo, Korea) was performed after at least 8 hours of bed rest before the test. After the patient lay in the supine position for 10 minutes, the test was performed for 20 minutes at a $70^{\circ}$ tilt. Patients who showed symptoms, such as dizziness, visual disturbance, or nausea, as well as those with atypical symptoms, such as headache and arm and leg pain or weakness during testing were considered positive. ${ }^{10)}$ If patients show negative result, repeat the HUT with pharmacological provocation. At the 2013, we changed protocols of HUT. During 2008-2013, isoproterenol was used for the pharmacological provocation, but after 2013, we changed it to nitroglycerine. Other than that, other protocols are same during our study periods.

VVS was classified by cardioinhibition, vasodepressor, mixed type VVS using previously defined diagnostic criteria. ${ }^{1)}$

TCD (Pioneer System TC 2020, Nicolet Biomedical Inc., Madison, WI, USA) was on the middle cerebral artery of patients, and systolic, diastolic, and mean cerebral artery blood flow were used to calculate the pulsatility index (PI) using the following formula: $\mathrm{PI}=\left(\mathrm{V}_{\text {systol }}-\mathrm{V}_{\text {diastol }}\right) / \mathrm{Vmean}$

\section{Autonomic function tests}

The CASS is calculated using sudomotor index, adrenergic index, cardiovascular heart rate index. And each index is calculated by following test.

The quantitative sudomotor axon reflex test (QSART) (Q-sweat, WR Medical Electronics Co., Maplewood, MN, USA) is the test evaluates the function of the postganglionic sympathetic sudomotor axon. ${ }^{11)}$ From the test, we could get the amount of sweating and latencies from 3 sites (forearm, proximal leg, distal leg). ${ }^{12,13)}$ And from that results, sudomotor index could be calculated. ${ }^{14)}$ The Valsalva test evaluates the sympathetic adrenergic and parasympathetic functions using heart rate responses. From the test, Valsalva ratio is calculated by dividing the highest heart rate by the lowest. ${ }^{14}$ ) The heart rate response to deep breathing was used to determine the cardiac parasympathetic function through the vagal nerve. For the test, lie down the patient on the bed and instructed to inhale and exhale for 5 seconds each for 6 times. After testing, the cardiovascular heart rate index is calculated. ${ }^{14)}$

From the previous study, it was challenging to quantifying the severity of autonomic dysfunction. For this reason, we performed autonomic function testing and calculate composite autonomic severity score (CASS). The CASS is the total sum of the sudomotor index, adrenergic index, cardiovascular heart rate index as a protocol. ${ }^{13)}$ Cardiovascular heart rate index is calculated by using Valsalva ratio and heart rate response to deep breathing. Adrenergic score is calculated from the Valsalva ratio and HUT results. From the total sum of cardiovascular heart rate index, adrenergic score, and sudomotor index, CASS is calculated. CASS ranges from 0 to 10 . In which a score between $0-3$ is classified as mild, 4-7 as moderate, and $8-10$ as severe. ${ }^{14)}$ From CASS, we could objectively quantitate autonomic dysfunc. tion.

\section{Statistical analysis}

All statistical analyses were performed using the PASW statics ver. 18.0 (SPSS Inc., Chicago, IL, USA). Mean values between the 2 groups were compared the independent $t$ test, and chi-square 
test was used for comparisons of categorical data. Statistical significance was defined as a $P<0.05$.

\section{Ethics statement}

This study was approved by the Institutional Review Board of Daegu Fatima Hospital (approval number: DFE20ORIO087).

\section{Results}

\section{Patient's characteristics}

The mean age of patients in our study was 13.8 years ranging from 8 to 18 years, with a sex ratio of 18 boys (27.3\%) to 48 girls (72.7\%). Forty-one patients, whose mean age was 14.1 years with a sex ratio of 8 boys (19.5\%) to 33 girls (80.5\%), were diagnosed with mild autonomic dysfunction. The remaining 25 patients of our study, whose mean age was 13.4 years with a sex ration of 10 boys (40.0\%) to 15 girls (60.0\%), were diagnosed with moderate autonomic dysfunction. We found no statistical differences between the 2 groups in sex distribution, body weight, height, body mass index, diastolic blood pressure, and heart rate (Table 1). In laboratory finding, complete blood cell count, electrolyte profile, thyroid function test, and $\mathrm{N}$ terminal pro B type natriuretic peptide shows no statistically difference between the 2 groups.

\section{Triggers and prodromal symptoms}

Among our 66 patients with VVS, we identified the following triggers: prolonged standing $(n=47)$, postural change $(n=14)$, bowel movement $(n=2)$, exercise $(n=1)$, stress $(n=1)$, and unknown $(n=1)$. We found no statistical differences in trigger cause between the 2 subgroups $(P=0.172)$ (Fig. 1$)$.
In the study group, some patients presented multiple prodro mal symptoms. The symptoms presented in our study were dizziness $(n=34)$, vision-related $(n=34)$, nausea/vomiting $(n=$ $5)$, weakness on extremity $(n=3)$, and headache $(n=1)$; no symptoms were reported in 3 cases. There were no differences in prodromal symptoms between our 2 groups $(P=0.149)$.

\section{HUT results}

All 66 patients in our study were positive on the HUT. Thirty patients were positive on HUT without using any medication, while 36 patients were positive on HUT after using medications such as isoproterenol or nitroglycerin. Among the 41 patients with mild autonomic dysfunction, 24 had vasodepressor VVS and 17 had mixed VVS. Among the 25 patients with moderate autonomic dysfunction, vasodepressor VVS was diagnosed in 15 and mixed VVS was diagnosed in 10 . We found no instances of cardioinhibitory VVS in our study. Furthermore, there were no statical differences in type of VVS between the 2 groups $(P=$

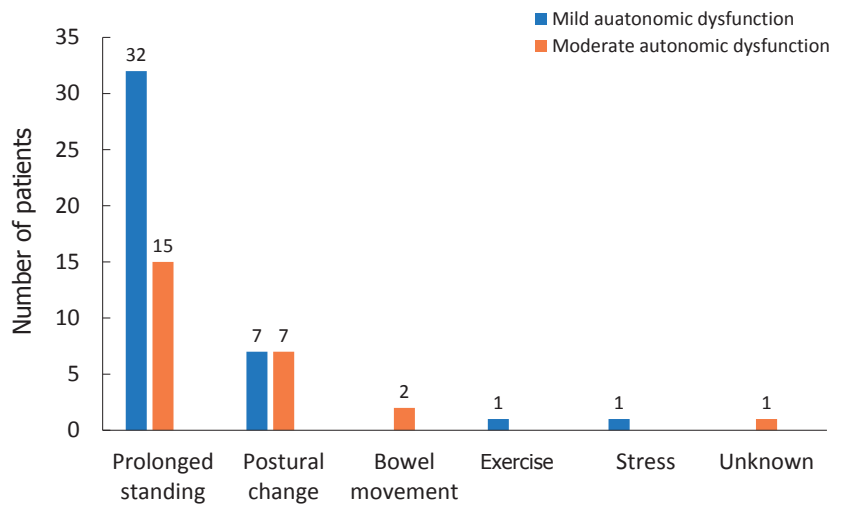

Fig. 1. Triggers of vasovagal syncope.

Table 1. Patient characteristics by study group

\begin{tabular}{|c|c|c|c|c|}
\hline Characteristic & $\begin{array}{l}\text { Total patients } \\
(\mathrm{n}=66)\end{array}$ & $\begin{array}{l}\text { Mild autonomic dysfunction } \\
\qquad(\mathrm{n}=41)\end{array}$ & $\begin{array}{l}\text { Moderate autonomic dysfunction } \\
\qquad(\mathrm{n}=25)\end{array}$ & $P$ value \\
\hline Age (yr) & $13.8 \pm 1.76$ & $14.1 \pm 1.61$ & $13.4 \pm 0.60$ & 0.575 \\
\hline \multicolumn{5}{|l|}{ Sex } \\
\hline Male & 18 & 8 & 10 & 0.070 \\
\hline Female & 48 & 33 & 15 & \\
\hline Type of vasovagal syncope & & & & 0.280 \\
\hline Cardioinhibition & 0 & 0 & 0 & \\
\hline Vasodepressed & 39 & 24 & 15 & \\
\hline Mixed & 27 & 17 & 10 & \\
\hline Height (cm) & $163.0 \pm 6.41$ & $163.0 \pm 5.87$ & $163.0 \pm 7.35$ & 0.275 \\
\hline Body weight (kg) & $54.9 \pm 11.97$ & $53.8 \pm 10.03$ & $56.8 \pm 14.66$ & 0.258 \\
\hline Body mass index $\left(\mathrm{kg} / \mathrm{m}^{2}\right)$ & $20.6 \pm 3.94$ & $20.2 \pm 3.44$ & $21.3 \pm 4.65$ & 0.250 \\
\hline Systolic blood pressure (mmHg) & $111.1 \pm 10.03$ & $109.4 \pm 11.01$ & $113.8 \pm 7.69$ & 0.033 \\
\hline Percentile & $55.56 \pm 27.22$ & $50.85 \pm 30.10$ & $63.28 \pm 19.91$ & 0.012 \\
\hline Diastolic blood pressure $(\mathrm{mmHg})$ & $63.8 \pm 8.08$ & $64.0 \pm 8.37$ & $63.3 \pm 7.73$ & 0.568 \\
\hline Percentile & $53.67 \pm 21.72$ & $47.78 \pm 25.23$ & $63.32 \pm 7.73$ & 0.412 \\
\hline Heart rate (frequency/min) & $84.9 \pm 11.67$ & $85.9 \pm 10.44$ & $83.3 \pm 13.52$ & 0.455 \\
\hline
\end{tabular}

Values are presented as number or mean \pm standard deviation or number.

Mild and moderate groups were classified according to composite autonomic severity score.

Boldface indicates a statistically significant difference with $P<0.05$. 
0.280) (Table 1).

\section{TCD results}

TCD was conducted in 66 patients of our study group. Diastolic blood flow, mean blood flow, and PI were measured by TCD in all patients before administration and during positive response on the HUT. Prior to testing, our study group had a mean diastolic blood flow of $53.85 \mathrm{~cm} / \mathrm{sec}$, mean blood flow of $74.39 \mathrm{~cm} / \mathrm{sec}$, and PI of 0.71 compared to a mean diastolic blood flow of $16.76 \mathrm{~cm} / \mathrm{sec}$, mean blood flow of $38.17 \mathrm{~cm} / \mathrm{sec}$, and PI of 1.51 when the positive response on the HUT. Diastolic blood flow and mean blood flow decreased by $68.7 \%$ and $46.7 \%$, respectively, while PI increased by $112.68 \%$ when patients showed symptoms.

Patients with mild autonomic dysfunction showed a mean diastolic blood flow of $54.83 \mathrm{~cm} / \mathrm{sec}$, mean blood flow of $75.88 \mathrm{~cm} / \mathrm{sec}$, and PI of 0.70 , whereas patients with moderate autonomic dysfunction showed a mean diastolic blood flow of $52.20 \mathrm{~cm} / \mathrm{sec}$, mean blood flow of $71.96 \mathrm{~cm} / \mathrm{sec}$, and PI of 0.73 . We found no significant differences in mean diastolic and mean blood flow rates between patients with mild and moderate autonomic dysfunction $(P>0.05)$. Moreover, the value of delta score between baseline and syncope (baseline value - syncope value) shows no statistical difference (Table 2).

\section{Autonomic function test results and severity of symptoms}

All 66 patients underwent autonomic function test. Mild autonomic dysfunction group shows mean Valsalva ratio of 2.03, heart rate response to deep breathing of 18.30 , cardiovascular index of 0.20 , adrenergic index of 1.17 , and sudomotor index of 0.95 . Moderate autonomic dysfunction group shows mean Valsalva ratio of 2.02, heart rate response to deep breathing of 20.28 , cardiovascular index of 0.32 , adrenergic index of 2.20 , and sudomotor index of 2.00. From this result, cardiovascular index and adrenergic index shows statistically meaningful $(P=$ 0.033 and $P<0.001$, respectively) (Table 3).

\section{Treatment outcomes and prognosis}

Before follow-up, all patients received patient education, such as encouraging lifestyle modifications and increased water intake. We teach patients to stand up slowly after lying down and take water approximately $2 \mathrm{~L} /$ day. And 39 patients take oral medicine in addition to education and lifestyle modification. The types of oral medications prescribed were propranolol $(n=29)$, midodrine $(n=9)$, propranolol and midodrine $(n=1)$. For the treatment, we could not compare the mild and moderate autonomic dysfunction group. As will be described later, biases are reflected so it is worthless to compare the 2 groups.

Patients were followed-up for an average of 4.6 months, and we found no significant differences in symptoms according to medication type and degree of autonomic dysfunction. After

Table 2. Transcranial Doppler results by study group

\begin{tabular}{lcc}
\hline Variable & $\begin{array}{c}\text { Mild autonomic dysfunction } \\
(\mathrm{n}=41)\end{array}$ & $\begin{array}{c}\text { Moderate autonomic dysfunction } \\
(\mathrm{n}=25)\end{array}$ \\
\hline Baseline & & 5 value \\
Diastolic blood flow (cm/sec) & $54.83 \pm 15.17$ & $52.20 \pm 12.46$ \\
Mean blood flow (cm/sec) & $75.88 \pm 21.01$ & $71.96 \pm 17.11$ \\
Pulsatility index & $0.70 \pm 0.15$ & $0.73 \pm 0.12$ \\
Syncope & & 0.722 \\
Diastolic (cm/sec) & $16.98 \pm 7.30$ & $16.40 \pm 7.07$ \\
Mean (cm/sec) & $38.44 \pm 10.21$ & $38.72 \pm 8.60$ \\
Pulsatility index & $1.54 \pm 0.49$ & $1.47 \pm 0.37$ \\
Delta score $(\Delta)$ between baseline and syncope $(\Delta=$ baseline-syncope) & & 0.798 \\
$\quad$ Diastolic blood pressure & $37.85 \pm 12.52$ & $35.80 \pm 13.12$ \\
Mean blood pressure & $37.44 \pm 13.46$ & $34.24 \pm 11.47$ \\
Pulsatility index & $0.84 \pm 0.46$ & $0.73 \pm 0.41$ \\
\hline
\end{tabular}

Values are presented as mean \pm standard deviation.

Mild and moderate groups were classified according to composite autonomic severity score.

Table 3. Autonomic function test results by study group

\begin{tabular}{lccc}
\hline Variable & $\begin{array}{c}\text { Mild autonomic dysfunction } \\
(\mathrm{n}=41)\end{array}$ & $\begin{array}{c}\text { Moderate autonomic dysfunction } \\
(\mathrm{n}=25)\end{array}$ & $P$ value \\
\hline Valsalva ratio & $2.03 \pm 0.41$ & $2.02 \pm 0.30$ & 0.230 \\
Heart rate response to deep breathing & $18.30 \pm 5.22$ & $20.28 \pm 6.94$ & 0.098 \\
Cardiovascular index & $0.20 \pm 0.40$ & $0.32 \pm 0.56$ & 0.033 \\
Adrenergic index & $1.17 \pm 0.50$ & $2.20 \pm 1.04$ & $<0.001$ \\
Sudomotor index (QSART) & $0.95 \pm 0.71$ & $2.00 \pm 1.04$ & 0.05 \\
\hline
\end{tabular}

Values are presented as mean \pm standard deviation.

QSART, quantitative sudomotor axon reflex test.

Mild and moderate groups were classified according to composite autonomic severity score.

Boldface indicates a statistically significant difference with $P<0.05$. 
Table 4. Treatment outcomes and patient prognosis

\begin{tabular}{lccc}
\hline Variable & $\begin{array}{c}\text { Mild autonomic } \\
\text { dysfunction } \\
(\mathrm{n}=\mathbf{4 1})\end{array}$ & $\begin{array}{c}\text { Moderate } \\
\text { autonomic } \\
\text { dysfunction } \\
(\mathrm{n}=25)\end{array}$ & $P$ value \\
\hline Treatment & 10 & 17 & $\mathbf{0 . 0 0 6}$ \\
Education & 31 & 8 & \\
Education + medication & 19 & 19 & 0.154 \\
During follow-up & 21 & 5 & \\
No symptoms & 1 & 1 & \\
Symptoms & & & \\
Follow-up loss & 19 & & \\
\hline
\end{tabular}

Mild and moderate groups were classified according to composite autonomic severity score.

Boldface indicates a statistically significant difference with $P<0.05$.

excluding 2 patients lost to follow-up, 38 (59.4\%) had no symptoms, while 26 (40.6\%) developed symptoms. The types of symptoms included at least 1 episode of dizziness $(n=23), 1$ episode of syncope $(n=2)$, and 2 or more episodes of syncope $(n=1)$ (Table 4).

\section{Discussion}

Syncope is a relatively common problem in adolescence, up to 15\%-50\% experience more than once before grown up. ${ }^{4,15,16)}$ Among the syncope patients, VVS is the most common and it is associated with autonomic dysfunction. ${ }^{17)}$ The main purpose of this study was to differentiate VVS patients according to their degree of autonomic dysfunction and analyze the difference between the 2 groups. In conclusion, we found no difference between the 2 groups, and no further evaluations are needed to classify the severity of autonomic dysfunction.

In this study, we performed laboratory test, ECG, HUT, TCD, and autonomic function tests for syncope diagnosis and severity assessment. It was previously shown that HUT is useful in diagnosis of syncope. ${ }^{1)}$ During HUT, the blood pressure and heart rate are monitored to evaluate hypotension, bradycardia, and tachycardia. In addition, TCD was given to evaluate cerebral blood flow. In our study group, we found significant decreases in the diastolic (68.9\%), mean (48.7\%) blood flows, and a significant increase in PI (112.64\%) at the time of a positive result in HUT compared to baseline.

Except for systolic blood pressure, we found no other differences in measures between mild and moderate autonomic dysfunction in our study. Comparisons between blood test results, TCD, prodromal symptoms, trigger factors, and followup outcomes revealed no differences between the 2 subgroups. In fact, only systolic blood pressure was slightly higher in the moderate autonomic dysfunction group by approximately 4 $\mathrm{mmHg}$ on average. Although our analysis indicated a statistically significant difference $(P=0.033)$, we consider that the finding may be meaningless because such blood pressure difference between the 2 groups would not be expected to cause a physiolo- gical difference in autonomic function.

Syncope is related with autonomic dysfunction, and conventionally, HUT is most commonly used for the diagnoses of VVS. However, HUT alone cannot determine the severity of autonomic dysfunction, and the etiology of approximately $50 \%$ $75 \%$ of cases remains unknown. ${ }^{18)}$ In such challenging cases, autonomic function tests can play a key role in the diagnosis and evaluation of the severity of autonomic dysfunction.

From the previous study, they underwent autonomic function test for the diagnosis of syncope, but they did not differentiate degree of autonomic dysfunction. ${ }^{19,20)}$ Consequently, there were no attempts to quantifying the degree of autonomic dysfunction of VVS patients and compare the VVS patients by the degree of autonomic dysfunction.

From this point, we start this study. The hypothesis underlying our current study was that there would be differences in the type, nature, and test results of VVS in our study group depending on the degree of autonomic nerve dysfunction. However, subgroup analysis found no evidence of either clinical or laboratory differences in VVS between patients with mild or moderate autonomic dysfunction. This finding suggests that such differences in VVS are not detectable compared to other classification categories using the CASS system. In addition, it may be that clinical or other measured differences in VVS are offset by compensatory mechanisms.

As mentioned above, during follow-up, we did education alone and education with orally administered medication such as propranolol and midodrine. Propranolol lessens ventricular mechanoreceptor activation owing to its antisympathetic and negative inotropic effect. ${ }^{21)}$ Midodrine increases blood pressure by increasing peripheral vascular resistance with a modest reduction in heart rate. ${ }^{22)}$ Using the chi-square test, we found a significant difference between patients with mild and moderate autonomic dysfunction $(P=0.006)$; however, it not that meaningful result. Because we use medication to compare syncope frequency and severity, but some patients wanted to be medicated irrespective of these factors, whereas other were not medicated even when it was recommended. Thus, we believe that this statistical finding is because of such biases and does not reflect a true association.

Our study had several limitations. First, we retrospectively analyzed medical records, and seconds, the number of enrolled patients was relatively small. These factors limited our ability to identify differences in VVS depending on the degree of autonomic dysfunction VVS.

In conclusion, using test of autonomic function, we evaluated the degree of autonomic dysfunction in patients with VVS and compare the 2 groups. Despite reviewing records at our center from the past 13 years, our study group of 66 patients may have insufficient power to allow identifying and defining the relationship between mild and moderate autonomic dysfunction in patients with VVS. Despite this limitation, the current study, shows that the degree of autonomic dysfunction may not be affected by characteristics, findings, parameters, and prognosis 
of VVS. Furthermore, although a definite relationship between mild and moderate autonomic dysfunction in patients with VVS was not identified, we propose that further study involving multiple centers and greater patient recruitment would reveal the relationship between the degree of autonomic dysfunction and characteristics, finding and prognosis of VVS.

\section{Footnotes}

Conflicts of interest : No potential conflict of interest relevant to this article was reported.

ORCID:

Dong Won Lee (1) https://orcid.org/0000-0001-6907-8613

\section{References}

1. Brignole M, Moya A, de Lange FJ, Deharo JC, Elliott PM, Fanciulli A, et al. 2018 ESC Guidelines for the diagnosis and management of syncope. Eur Heart J 2018;39:1883-948.

2. Schraeder PL, Lathers CM, Charles JB. The spectrum of syncope. J Clin Pharmacol 1994;34:454-9.

3. Njemanze PC. Critical limits of pressure-flow relation in the human brain. Stroke 1992;23:1743-7.

4. Massin MM, Bourguignont A, Coremans C, Comté L, Lepage P, Gérard P. Syncope in pediatric patients presenting to an emergency department. J Pediatr 2004;145:223-8.

5. Benditt DG, van Dijk JG, Sutton R, Wieling W, Lin JC, Sakaguchi S, et al. Syncope. Curr Probl Cardiol 2004;29:152-229.

6. Freeman R, Chapleau MW, Testing the autonomic nervous system. Handb Clin Neurol 2013;115:115-36.

7. Low PA, Denq JC, Opfer-Gehrking TL, Dyck PJ, O'Brien PC, Slezak JM. Effect of age and gender on sudomotor and cardiovagal function and blood pressure response to tilt in normal subjects. Muscle Nerve 1997;20: 1561-8.

8. Medow MS, Merchant S, Suggs M, Terilli C, O'Donnell-Smith B, Stewart JM. Postural heart rate changes in young patients with vasovagal syncope. Pediatrics 2017;139:e20163189.
9. Adkisson WO, Benditt DG. Syncope due to autonomic dysfunction: diagnosis and management. Med Clin North Am 2015;99:691-710.

10. Gourishankar A, Belton MD, Hashmi SS, Butler IJ, Lankford JE, Numan MT. Demographic and clinical features of pediatric patients with orthostatic intolerance and an abnormal head-up tilt table test; a retrospective descriptive study. Pediatr Neonatol 2020;61:68-74.

11. Novak P. Quantitative autonomic testing. J Vis Exp 2011;(53):2502.

12. Low PA. Autonomic nervous system function. J Clin Neurophysiol 1993; 10:14-27.

13. Low PA. Laboratory evaluation of autonomic function. Suppl Clin Neurophysiol 2004;57:358-68.

14. Low PA. Composite autonomic scoring scale for laboratory quantification of generalized autonomic failure. Mayo Clin Proc 1993;68:748-52.

15. Sun BC, Emond JA, Camargo CA Jr. Characteristics and admission patterns of patients presenting with syncope to U.S. emergency departments, 1992-2000. Acad Emerg Med 2004;11:1029-34.

16. Ganzeboom KS, Colman N, Reitsma JB, Shen WK, Wieling W. Prevalence and triggers of syncope in medical students. Am J Cardiol 2003;91:1006$8, \mathrm{~A} 8$.

17. Jarjour IT. Postural tachycardia syndrome in children and adolescents. Semin Pediatr Neurol 2013;20:18-26.

18. Moya A. Tilt testing and neurally mediated syncope: too many protocols for one condition or specific protocols for different situations? Eur Heart J 2009;30:2174-6.

19. Sneddon JF, Counihan PJ, Bashir Y, Haywood GA, Ward DE, Camm AJ. Assessment of autonomic function in patients with neurally mediated syncope: augmented cardiopulmonary baroreceptor responses to graded orthostatic stress. J Am Coll Cardiol 1993;21:1193-8.

20. Tao C, Tang C, Chen S, Jin H, Du J. Autonomic nervous function in vasovagal syncope of children and adolescents. Neurosci Bull 2019;35: 937-40.

21. Sra JS, Murthy VS, Jazayeri MR, Shen YH, Troup PJ, Avitall B, et al. Use of intravenous esmolol to predict efficacy of oral beta-adrenergic blocker therapy in patients with neurocardiogenic syncope. J Am Coll Cardiol 1992;19:402-8.

22. Ward CR, Gray JC, Gilroy JJ, Kenny RA. Midodrine: a role in the management of neurocardiogenic syncope. Heart 1998;79:45-9.

How to cite this article: Lee HE, Lee DW. Vasovagal syncope with mild versus moderate autonomic dysfunction: a 13-year single-center experience. Clin Exp Pediatr 2022;65:47-52. https://doi.org/10.3345/cep.2021.00052 